\title{
Rare-earth high-entropy alloys with giant magnetocaloric effect
}

\author{
Y. Yuan ${ }^{1}$, Y. Wu ${ }^{1 *}$, X. Tong ${ }^{2,3}$, H. Zhang ${ }^{4}$, H. Wang ${ }^{1}$, X. J. Liu ${ }^{1}$, L. Ma ${ }^{5}$, H. L. Suo ${ }^{5}$, \\ Z. P. $\mathbf{L u}^{\mathbf{1}^{*}}$
}

${ }^{1}$ State Key Laboratory for Advanced Metals and Materials, University of Science and Technology Beijing, Beijing 100083, China

${ }^{2}$ Instrument and Source Division, Oak Ridge National Laboratory, TN, 37831, USA

${ }^{3}$ Department of Physics and Astronomy, University of Tennessee, Knoxville, TN, 37996, USA

${ }^{4}$ School of Materials science and Engineering, University of Science and Technology Beijing, Beijing 100083, China

${ }^{5}$ College of Materials Science and Engineering, Beijing University of Technology, Beijing 100124, China

\begin{abstract}
In this paper, we report the development of rare-earth high-entropy alloys (RE-HEA) with multiple principle elements randomly distributed on a single hexagonal close-packed (HCP) lattice. Our work demonstrated that it is the entropy, rather than other atomic factors such as enthalpy, atomic size and electronegativity, that dictates phase formation in the current rare-earth alloy system. The high configuration entropy stabilized the crystalline structure from phase transformation during cooling, whereas a second-order magnetic phase transition occurred at its Neel temperature. The quinary RE-HEA exhibited a small magnetic hysteresis and the largest refrigerant capacity (about $627 \mathrm{~J} \mathrm{~kg}^{-1}$ at the $5 \mathrm{~T}$ magnetic field) reported to date, along with respectable mechanical properties. Our analysis indicates that the strong chemical disorder resulted from the high configuration entropy makes magnetic ordering in the HEA difficult, thus giving rise to a sluggish magnetic phase transition and enhanced magnetocaloric effect. Our findings evidenced that RE-HEAs have great potential to be used as magnetic refrigerants and the alloy-design concept of HEAs can be employed to develop novel high-performance magnetocaloric materials.
\end{abstract}

*Correspondence and requests for materials should be addressed to Y. W. (email: wuyuan@ustb.edu.cn) or Z. P. L. (email:luzp@ustb.edu.cn) 


\section{Introduction}

Compared with conventional gas refrigerants, magnetic refrigerants based on the magnetocaloric effect (MCE) have advantages of being both highly efficient and environmental friendly [1-4]. The magnetocaloric effect can be characterized by the field-induced entropy change $\left(\Delta \mathrm{S}_{\mathrm{M}}\right)$ due to the alignment of its magnetic spins that occurs under an external magnetic field [5]. According to the involved magnetic phase transitions, magnetic refrigerants can be divided into two categories, i.e., the first and the second order magnetic phase transition materials. Materials in the former category usually show large MCE in a narrow temperature range, but their large thermal and magnetic hysteresis and easy occurrence of cracking and fatigue limited their widespread use as magnetic refrigerators [6]. In contrast, the MCE materials in the second category normally possess gradual and continuous magnetization variation, exhibiting broader peaks with no thermal and magnetic hysteresis. Currently, this type of MCE materials is considered to be an optimal choice for magnetic refrigerants [7]. Up to now, however, very few alloys in this specific category simultaneously having large magnetic entropy change and refrigerant capacity (RC) have been developed and fabricated. Exploring novel alloys concurrently possessing large magnetic entropy change and $\mathrm{RC}$ through innovative routes is paramount for enabling practical applications of magnetic refrigerants.

In recent years, a new alloy-design concept, termed as high-entropy alloys (HEAs), was proposed [8-10]. Generally, HEAs contain multiple principal elements in 
an equimolar or near-equimolar ratio, which induces formation of disordered solid-solution phases with simple structures due to the high entropy of mixing, such as body-centered cubic (BCC), face-centered cubic (FCC) or hexagonal close-packed (HCP) structures. Formation of ordered crystalline intermetallic phases that often contain structurally complex giant unit cells is usually suppressed [11]. The HEA crystallographic structure is characterized by a topologically ordered lattice with an exceedingly high chemical disorder. Such a configurational disorder in MCE materials might hinder thermal motion of magnetic atoms or ions due to the so-called entropy stabilization, which could result in increased heat flow from the ambient environment and lead to large MCE. Rare-earth (RE) elements are known to possess unique magnetocaloric effects, therefore, it is interesting and imperative, both scientifically and technologically, to apply the HEA concept into developing high-performance RE-MCE materials. Recently, Feuerbacher et al [12] first reported the development of a HCP HEA using pure RE metals, suggesting that our approach is feasible. In this paper, we report design and fabrication of several RE-HEAs which simultaneously have large MCE and RC, and the underlying mechanisms will also be explored. 


\section{Experimental}

Alloys with a nominal composition of $\mathrm{Gd}_{20} \mathrm{Dy}_{20} \mathrm{Er}_{20} \mathrm{Ho}_{20} \mathrm{~Tb}_{20}, \mathrm{Gd}_{25} \mathrm{Er}_{25} \mathrm{Ho}_{25} \mathrm{~Tb}_{25}$, $\mathrm{Dy}_{25} \mathrm{Er}_{25} \mathrm{Ho}_{25} \mathrm{~Tb}_{25}$ and $\mathrm{Er}_{33.33} \mathrm{Ho}_{33.33} \mathrm{~Tb}_{33.34}$ were prepared by arc-melting a mixture of high-purity Gd (99.9\%), Dy (99.9\%), Er (99.9\%), Ho (99.9\%) and Tb (99.9\%) in a Ti-gettered high-purity argon atmosphere. The ingots were re-melted at least six times to ensure chemical homogeneity and subsequently drop-cast into a copper mold with a dimension of $\Phi 10 \mathrm{~mm} \times 60 \mathrm{~mm}$. Phase constitutions were identified by X-ray diffraction (XRD) using $\mathrm{Cu}-\mathrm{K} \alpha$ radiation. Chemical composition was analyzed by energy dispersive spectrometer (EDS) in a scanning electron microscopy (SEM) with field emission using a Zeiss Supra55 operated at $15 \mathrm{kV}$. SEM, TEM (transmission electron microscopy) and STEM (scanning transmission electron microscopy) were employed to characterize the microstructures. The SEM samples were sequentially ground and mechanically polished using $0.04 \mu \mathrm{m}$ silica suspensions. The TEM samples were first mechanically ground to $\sim 20 \mu \mathrm{m}$ thick and then ion-milled at $-30^{\circ} \mathrm{C}$ under liquid-nitrogen to avoid oxidation. Temperature and field dependences of magnetization as well as specific heat were measured by a physical property measurement system (PPMS) from Quantum Design Company. 


\section{Results}

\subsection{Microstructure}

Figure 1a shows $\mathrm{XRD}$ patterns of the as-cast $\mathrm{Gd}_{20} \mathrm{Dy}_{20} \mathrm{Er}_{20} \mathrm{Ho}_{20} \mathrm{~Tb}_{20}$, $\mathrm{Gd}_{25} \mathrm{Er}_{25} \mathrm{Ho}_{25} \mathrm{~Tb}_{25}, \mathrm{Dy}_{25} \mathrm{Er}_{25} \mathrm{Ho}_{25} \mathrm{~Tb}_{25}$, and $\mathrm{Er}_{33.33} \mathrm{Ho}_{33.33} \mathrm{~Tb}_{33.34}$ alloys. For the quinary $\mathrm{Gd}_{20} \mathrm{Dy}_{20} \mathrm{Er}_{20} \mathrm{Ho}_{20} \mathrm{~Tb}_{20}$ alloy, all the XRD peaks can be indexed to a HCP structure. The corresponding SEM image of this alloy is shown in Fig. 1b in which irregular-shaped grains are seen, indicating formation of a mostly single crystalline phase. While for the quaternary $\mathrm{Gd}_{25} \mathrm{Er}_{25} \mathrm{Ho}_{25} \mathrm{~Tb}_{25}$ and $\mathrm{Dy}_{25} \mathrm{Er}_{25} \mathrm{Ho}_{25} \mathrm{~Tb}_{25}$ alloys, and ternary $\mathrm{Er}_{33.33} \mathrm{Ho}_{33.33} \mathrm{~Tb}_{33.34}$ alloy, another crystalline phase with a trigonal (TRI) structure was found in addition to the HCP phase, indicating that configuration entropy of these alloys is insufficient to induce formation of a single phase. The actual composition was determined to be $\mathrm{Gd}_{19.5} \mathrm{Dy}_{21.1} \mathrm{Er}_{19.3} \mathrm{Ho}_{20} \mathrm{~Tb}_{20.1}$ (in atomic percent) with $0.5 \%$ uncertainty from the EDS measurements, which is in good agreement with the target. XRD patterns of $\mathrm{Gd}_{20} \mathrm{Dy}_{20} \mathrm{Er}_{20} \mathrm{Ho}_{20} \mathrm{~Tb}_{20}$ as a function of temperature from $20 \mathrm{~K}$ to room temperature are shown in Fig. 1b. Clearly, the XRD patterns stayed almost the same at different temperatures, suggesting neither structural change nor structure-induced phase transformation during cooling down to $20 \mathrm{~K}$. Nevertheless, the XRD peaks were found to be quite broad, as compared with those in conventional alloys, which may be due to the distorted HCP lattice resulted from the high configurational entropy [12]. Based on Vegard's rule of mixture, the lattice parameter can be estimated by $a_{m i x}=\sum_{i} c_{i} a_{i}$, which is valid for completely random mixing of the elements ( $c_{i}$ is atomic fraction and $a_{i}$ is lattice parameter of the element i). As 
shown in Table 1 which summarizes lattice parameters $a$ and $c$, atomic radius $\gamma$, Curie temperature $T_{c}$, Neel temperature $T_{N}$ and total angular momentum $\mathrm{J}$ of pure constituent $\mathrm{RE}$ elements and the investigated $\mathrm{Gd}_{20} \mathrm{Dy}_{20} \mathrm{Er}_{20} \mathrm{Ho}_{20} \mathrm{~Tb}_{20}$ HEA. The theoretical lattice parameter of the current RE-HEA was calculated to be a=3.594 $\AA$ and $c=5.666 \AA$, which is in good agreement with experimental values (i.e., a=3.599 $\AA$ and $\mathrm{c}=5.678 \AA$ ), suggesting that the RE constituent elements are likely randomly mixed in the current HCP lattice (Table 1).

A STEM image of the $\mathrm{Gd}_{20} \mathrm{Dy}_{20} \mathrm{Er}_{20} \mathrm{Ho}_{20} \mathrm{~Tb}_{20} \mathrm{HEA}$ is shown in Fig. 2a. No distinct features due to composition variation, precipitation of secondary phases, or dendrite formation were observed, further manifesting that the current multicomponent RE-HEA mainly consists of a homogeneous single phase structure. The corresponding selected area electron diffraction (SAED) patterns along different zone axes are shown in Figs. 2b-2d. The diffraction pattern along the [0001] zone axis has an m symmetry, which corresponds to the point group $6 / \mathrm{mmm}$ in a hexagonal system. The (0001) reflection is visible along the [2110] zone axis, but essentially diminishes when tilting to [0110], which indicates the presence of a $6_{3}$ screw axis parallel to [0001]. Therefore, the space group can be identified as $\mathrm{P}_{3} / \mathrm{mmc}$, which is similar to the observation reported in Ref. 12.

\subsection{Magnetization behavior}

Temperature dependence of zero-field-cooling (ZFC) magnetizations under various magnetic fields in the temperature range between 50 and $300 \mathrm{~K}$ for the 
$\mathrm{Gd}_{20} \mathrm{Dy}_{20} \mathrm{Er}_{20} \mathrm{Ho}_{20} \mathrm{~Tb}_{20} \mathrm{HEA}$ is shown in Fig. 3. Under a low magnetic field $(\mathrm{H})$ of 0.3 T, a small kink was observed and the Neel temperature can be determined as $T_{N}=186$ K using Gaussian fit(see the inset of Fig. 3). This temperature corresponds to a magnetic ordering transition from paramagnetic (PM) to a long-range ordered antiferromagnetic (AFM) state, which is similar to the observation reported by Luznik et al [13]. In addition, it is found that the magnetization increases with further decrease of temperature below $\mathrm{T}_{\mathrm{N}}$, which is possibly related to another first-order magnetic transition from an AFM to FM (ferromagnetic) state. With increasing magnetic fields, the singularity around $\mathrm{T}_{\mathrm{N}}$ gradually became weaker and eventually disappeared at the $3 \mathrm{~T}$ field, indicating the suppression of the PM-AFM transition due to the field-induced metamagnetic transition from AFM to FM states. Finally, only a sluggish PM-FM transition was observed when the magnetic field increased to 3T.

The magnetization isotherms $\mathrm{M}-\mu_{0} \mathrm{H}$ in the temperature range from 90 to $230 \mathrm{~K}$ with an interval of $5 \mathrm{~K}$ as a function of magnetic field (up to a maximum of $5 \mathrm{~T}$ ) for the $\mathrm{Gd}_{20} \mathrm{Dy}_{20} \mathrm{Er}_{20} \mathrm{Ho}_{20} \mathrm{~Tb}_{20}$ HEA are shown in Fig. 4a. To ensure the same phase for the integrity of the data, the sample was re-heated to the room temperature and then cooled to the next temperature for the $\mathrm{M}-\mu_{0} \mathrm{H}$ measurements. At low temperatures, the magnetization increases rapidly at low fields and tends to saturate with further increasing of magnetic field, corresponding to the typical FM behavior. However, it is interesting to note that the magnetization at the temperatures below $T_{N}$ increases linearly with the field at first, and then exhibits a sharp jump when the field is higher than a critical value. Meanwhile, a weak magnetic hysteresis was also observed in this 
temperature range (Fig. 4b). These phenomena confirm the presence of the AFM components in this temperature range and occurrence of metamagnetic transition from AFM to FM states $[14,15]$.

\subsection{Magnetic entropy change and refrigerant capacity}

The corresponding entropy change $\left|\Delta S_{M}\right|$ associated with the $\mathrm{H}$ variation can be calculated by integrating the Maxwell relation [16], i.e., $\Delta \mathrm{S}_{\mathrm{M}}(\mathrm{T}, \mathrm{H})=\int_{H \min }^{H \max }\left(\frac{\partial M}{\partial T}\right)_{H} d H$, where $H_{\min }$ and $H_{\max }$ represent the initial and final value of magnetic field, respectively. The calculated curves of the magnetic entropy change at different fields are shown in Fig. 5. The peak magnetic entropy change $\left|\Delta s_{M}^{p k}\right|$ under the field change of $5 \mathrm{~T}$ reaches as high as $8.6 \mathrm{Jkg}^{-1} \mathrm{~K}^{-1}$ around $T_{N}$. In addition, a small positive $\Delta S_{M}$ value (i.e., inverse MCE) was seen in the temperature range just below $T_{N}$, which is due to the presence of the AFM components. With the increase of magnetic field, this positive $\Delta S_{M}$ gradually changes to negative value because of the field-induced metamagnetic transition from the AFM to FM state. Another point worth noting is that a broad hump on the $\Delta S_{M}$ curve is present around $120 \mathrm{~K}$ under low magnetic field changes, which is related to the magnetic transition from the AFM to FM state with decreasing temperature. For the high field of $5 \mathrm{~T}$, it is clear that the combination of the broad $\Delta S_{M}$ hump and the high $\Delta S_{M}$ peak around $\mathrm{T}_{\mathrm{N}}$ enables a large working temperature range.

The refrigerant capacity (RC) value has been considered as another important 
measure of how much heat is transferred between the hot and cold ends in an ideal refrigeration cycle. To thoroughly evaluate the RC of the current $\mathrm{Gd}_{20} \mathrm{Dy}_{20} \mathrm{Er}_{20} \mathrm{Ho}_{20} \mathrm{~Tb}_{20}$ RE-HEA, three different criteria were employed[17-19]: (a) the product of $\left|\triangle S_{M}^{\text {peak }}\right| \times \delta T_{\mathrm{FWHM}}$ (denoted as RC-1), where $\delta T_{\mathrm{FWHM}}=T_{\text {hot }}-T_{\text {cold }}$, corresponds to the full-width at the half-maximum of the $\triangle S_{M}(T)$ curve; it is usually assumed that $\delta T_{\mathrm{FWHM}}$ coincides with the temperature span of the thermodynamic cycle being $T_{\text {hot }}$ and $T_{\text {cold }}$ the hot and cold ends, respectively; (b) the value of the integral area under the $\triangle S_{M}(T)$ curve between $T_{\text {hot }}$ and $T_{\text {cold }}$ (denoted as RC-2, as the hatched area shown in the inset of Fig.5), and (c) the maximum value of $\triangle S_{M} \times \delta$ T below the $\triangle S_{M}(T)$ curve (denoted as RC-3). Figure 6 shows the calculated values of RC-1, RC-2 and RC-3 as a function of magnetic field. For a magnetic field of $5 \mathrm{~T}$, the $\mathrm{RC}-1, \mathrm{RC}-2$ and $\mathrm{RC}-3$ value of the $\mathrm{Gd}_{20} \mathrm{Dy}_{20} \mathrm{Er}_{20} \mathrm{Ho}_{20} \mathrm{~Tb}_{20}$ HEA was determined to be 895,627 and $437 \mathrm{~J} \mathrm{~kg}^{-1}$, respectively. Note that RC-2 will be used for comparison with other materials and for further discussion hereafter. To our knowledge, the RC values of the current RE-HEA is the largest among all reported magnetic refrigeration materials in the same temperature range (see Table 2). This giant $\mathrm{RC}$ value is due to the combination of the successive $\Delta S_{M}$ peaks which results in the significant expansion of the working temperature range. 


\section{Discussion}

\subsection{Phase formation}

To evaluate the effect of mixing entropy on phase stability in the current RE-HEA, the parameter $\boldsymbol{\Omega}$ was calculated [20]:

$\boldsymbol{\Omega}=\frac{T_{m} \Delta S_{\text {mix }}}{\Delta H_{\text {mix }}}$

Where $\mathrm{T}_{\mathrm{m}}, \triangle \mathrm{S}_{\text {mix }}$ and $\triangle H_{\text {mix }}$ are the average melting temperature, the entropy and enthalpy of mixing of the alloy system, respectively, which are calculated separately as:

$T_{m}=\sum_{i=1}^{n} c_{i}\left(T_{m}\right)_{i}$

$\triangle S_{\text {mix }}=-\mathrm{R} \sum_{i=1}^{n}\left(c_{i} \ln c_{i}\right)$

$\Delta \mathrm{H}_{\mathrm{mix}}=\sum_{i=1, i \neq \mathrm{j}}^{n} \Omega_{i j} c_{i} c_{j}=4 \sum_{i=1, i \neq \mathrm{j}}^{n} \Delta H_{i j}^{\text {mix }} c_{i} c_{j}$

where $\left(\mathrm{T}_{\mathrm{m}}\right)_{\mathrm{i}}$ is the melting point of the $\mathrm{i}^{\text {th }}$ element, $\mathrm{R}\left(=8.314 \mathrm{~J} \mathrm{~K}^{-1} \mathrm{~mol}^{-1}\right)$ is the gas constant, $\mathrm{c}_{\mathrm{i}}$ is the molar ratio and $\Delta H_{i j}^{m i x}$ is the enthalpy of mixing between the $\mathrm{i}^{\text {th }}$ and $\mathrm{j}^{\text {th }}$ elements. It is considered that the entropy term dominate over the enthalpy and stabilizes the solid solution phase when $\boldsymbol{\Omega}$ is larger than 1.1 [20]. In the current equimolar alloys, the entropy of mixing can be calculated to be $13.38 \mathrm{~J} / \mathrm{K} \mathrm{mol}$ $(=1.61 \mathrm{R})$ for the quinary alloy $\mathrm{Gd}_{20} \mathrm{Dy}_{20} \mathrm{Er}_{20} \mathrm{Ho}_{20} \mathrm{~Tb}_{20}, 11.53 \mathrm{~J} / \mathrm{K}$ mol (=1.386R) for the quaternary alloys $\mathrm{Gd}_{25} \mathrm{Er}_{25} \mathrm{Ho}_{25} \mathrm{~Tb}_{25}$ and $\mathrm{Dy}_{25} \mathrm{Er}_{25} \mathrm{Ho}_{25} \mathrm{~Tb}_{25}$, and $9.14 \mathrm{~J} / \mathrm{K} \mathrm{mol}$ (=1.1R) for the ternary alloy $\mathrm{Er}_{33.33} \mathrm{Ho}_{33.33} \mathrm{~Tb}_{33.34}$. According to Yeh et al [9], the quinary alloy can be categorized as a high entropy alloy, whilst the rest can only be regarded as medium entropy alloy. Due to the similarity of the constituent elements, 
the heat of mixing for all alloys approaches zero. Therefore, the parameter $\boldsymbol{\Omega}$ is large and the entropy of mixing should predominate the thermodynamic landscape and induce formation of a single phase in all the rare-earth alloys. However, the single solid-solution phase was only found in the quinary alloy, indicating that entropy effect is critical in achieving a single phase structure in the current rare-earth system.

Based on Hume-Rothery rules, another parameter $\delta$ (i.e., the atomic radius difference) was proposed topologically to predict structural stability and phase formation in HEAs [21]:

$\delta=\sqrt{\sum_{i=1}^{n} c_{i}\left(1-r_{i} / \bar{r}\right)^{2}}$

where $r_{i}$ is the Goldschmidt atomic radius of the $i_{\text {th }}$ element. The parameter $\delta$ characterizes the atomic size mismatch, which reflects the local elastic strain and topological instability of the alloy system [22]. Using available data from various HEA systems, the criterion for forming simple solid solutions in HEAs upon rapid solidification was determined to $\delta<4 \%$. Due to the similar atomic radius of the constituent RE elements, it is clear that all four rare-earth alloys should form single phase because their $\delta$ value approaches zero.

To further estimate the effects of atomic size mismatch and lattice distortion in the current alloys, intrinsic residual strain was also assessed from atomic size and packing efficiency according to Ye et al. [23]:

$\varepsilon_{i}=\frac{\sum_{j=1}^{n} \omega_{i j} c_{j}}{\sum_{k=1}^{n} A_{i k} c_{k}}-\frac{4 \pi \eta}{N_{i} \sum_{k=1}^{n} A_{i k} c_{k}}$

where $\omega_{i j}$ is to quantify the local atomic packing efficiency and it can be expressed 
as $\omega_{i j}=2 \pi\left[1-\frac{\sqrt{\gamma_{\mathrm{i}}\left(\gamma_{\mathrm{i}}+2 \gamma_{\mathrm{j}}\right)}}{\gamma_{\mathrm{i}}+\gamma_{\mathrm{j}}}\right], A_{i k}$ is calculated by $A_{i k}=\frac{2 \pi \chi_{\mathrm{ik}}}{\left(\chi_{\mathrm{ik}}+1\right)^{2} \sqrt{\chi_{\mathrm{ik}}\left(\chi_{\mathrm{ik}}+2\right)}}$ and the atomic size ratio $\chi_{\mathrm{ik}}=\frac{\gamma_{\mathrm{i}}}{\gamma_{\mathrm{k}}}, c_{j}$ is the probability of having the atom $\mathrm{j}$ as the nearest neighbor of the central atom and it is taken as 0.2 in the HEA studied due to completely disordering arrangement. $\mathrm{N}$ is the coordination number and we consider it to be 12 since the space group is HCP according to the XRD result.

In Ye's study, solid solution is found to be stable at a root mean square (R.M.S.) residual strain $\left(\sqrt{<\varepsilon^{2}>}=\sqrt{\sum_{j=1}^{n} c_{j} \varepsilon_{j}^{2}}\right)$ lower than $5 \%$. The $\sqrt{<\varepsilon^{2}>}$ value is calculated to be $0.167 \%, 0.458 \%, 3 \%$ and $2 \%$ for $\mathrm{Gd}_{20} \mathrm{Dy}_{20} \mathrm{Er}_{20} \mathrm{Ho}_{20} \mathrm{~Tb}_{20}$, $\mathrm{Gd}_{25} \mathrm{Er}_{25} \mathrm{Ho}_{25} \mathrm{~Tb}_{25}, \mathrm{Dy}_{25} \mathrm{Er}_{25} \mathrm{Ho}_{25} \mathrm{~Tb}_{25}$ and $\mathrm{Er}_{33.33} \mathrm{Ho}_{33.33} \mathrm{~Tb}_{33.34}$, respectively. They are all located in the single solid-solution zone. From a view of strain energy, there is no apparent lattice straining effect and therefore giant MCE and RC can hardly be attributed to the lattice distortion of HEAs.

Furthermore, other HEA formation criteria, e.g., the electronegativity difference, $\triangle \chi[24]$ and valence electron concentration (VEC) $[25,26]$ were also examined for the current four rare-earth alloys. The value of $\triangle \chi$ is calculated to be $0.016,0.018$, 0.015 and 0.017 for $\mathrm{Gd}_{20} \mathrm{Dy}_{20} \mathrm{Er}_{20} \mathrm{Ho}_{20} \mathrm{~Tb}_{20}, \mathrm{Gd}_{25} \mathrm{Er}_{25} \mathrm{Ho}_{25} \mathrm{~Tb}_{25}, \mathrm{Dy}_{25} \mathrm{Er}_{25} \mathrm{Ho}_{25} \mathrm{~Tb}_{25}$ and $\mathrm{Er}_{33.33} \mathrm{Ho}_{33.33} \mathrm{~Tb}_{33.34}$, respectively. The VEC value is determined to be 3 for all alloys because each individual component $\mathrm{VEC}=3$ for all component elements.

As discussed above, all criteria suggest that a single phase structure would be presented in all four rare-earth alloys, which contradicts with the experimental findings and reveals a dominant effect of entropy on stabilizing phase formation. Nevertheless, the combination of extremely low $\delta$, nearly zero $\Delta \mathrm{H}_{\text {mix }}$ and low $\Delta \chi$ 
renders the $\mathrm{Gd}_{20} \mathrm{Dy}_{20} \mathrm{Er}_{20} \mathrm{Ho}_{20} \mathrm{~Tb}_{20}$ HEA a nearly ideal solid solution.

\subsection{Magnetic phase transition}

A summary of magnetic entropy changes and related magnetocaloric properties of the current quinary RE-HEA is given in Table 2, in comparison with those of typical MCE materials. Clearly, the currently developed RE-HEA with a single HCP phase shows high magnetic entropy change and the largest $\mathrm{RC}$ among the quaternary and ternary rare-earth alloys and other typical MCE materials, indicating beneficial contribution from the high configuration disorder and single solid solution phase structure in the quinary alloy.

As elaborated earlier, characteristics of magnetic phase transition would effectively affect practical applications of magnetic refrigerants. To reveal the nature of the magnetic transition, the Arrott plots derived from the $\mathrm{M}-\mu_{0} \mathrm{H}$ isotherms are plotted in Fig. 7. According to the Banerjee's criterion, a magnetic transition is considered as first-order when the Arrott plots exhibit a negative slope; otherwise, it is second-order [27]. It is evident that the slope is positive above $185 \mathrm{~K}$, suggesting the nature of a second-order PM-AFM transition. Nevertheless, a negative slope was observed from the plot below $185 \mathrm{~K}$, implying that a first-order transition from the AFM to FM state occurred at low temperatures [27].

To further clarify the nature of magnetic phase transition in the current RE-HEA, the zero magnetic field specific heat $\mathrm{C}_{\mathrm{p}}$ was measured and the result is shown in Fig. 
8.The $\mathrm{Gd}_{20} \mathrm{Dy}_{20} \mathrm{Er}_{20} \mathrm{Ho}_{20} \mathrm{~Tb}_{20}$ HEA exhibits a typical broad $\lambda$-like peak at the Neel temperature $T_{N}=186 \mathrm{~K}$, which is consistent with the result of magnetic measurements. Meanwhile, the $\lambda$ singularity in the specific heat at $T_{N}$ is similar to that of Gd [28] with a second-order transition, but different from $\mathrm{Gd}_{5} \mathrm{Si}_{2} \mathrm{Ge}_{2}$ which showed a first-order transition and a sharp peak in the specific heat [29], further confirming the nature of the second-order PM-AFM transition in the quinary RE-HEA. In addition, a broad hump was observed around $120 \mathrm{~K}$, which is likely attributed to the magnetic transition from the AFM to FM state, also in good agreement with the magnetic measurements.

In MCE materials, large hysteretic losses usually make magnetic refrigeration less efficient. In the current RE-HEA, only a small hysteresis was observed just below $\mathrm{T}_{\mathrm{N}}$. A summary of hysteretic loss values over the temperature is presented in Fig. 9. These hysteretic losses were determined by computing the hatched area inside each magnetization (M) versus field (H) loop (i.e., Fig. 4b). In the temperature ranges where the RC values were numerically integrated, the average hysteretic loss is about $7 \mathrm{~J} \mathrm{~kg}^{-1}$ for this HEA. Because this value represents the energy loss during cycling of the magnetic field, it must be taken into account as far as the usefulness of a magnetic refrigerant is considered. One typical way to evaluate the hysteresis loss of each alloy is to simply subtract it from the corresponding RC value. Subtraction of the average hysteresis from the corresponding RC values yielded an approximate value of $620 \mathrm{~J}$ $\mathrm{kg}^{-1}$ for the current RE-HEA (i.e., RC-2 as in section 3.3). On this basis, therefore, the quinary $\mathrm{Gd}_{20} \mathrm{Dy}_{20} \mathrm{Er}_{20} \mathrm{Ho}_{20} \mathrm{~Tb}_{20}$ HEA not only has large $\mathrm{RC}$ but also is highly 
refrigeration efficient, even better than that of the GdGeSi systems [29, 30].

Therefore, the currently developed quinary RE-HEAs have several advantages over conventional MCE materials. First, RC is the largest within the MCE materials as listed in Table 2 . The maximum magnetic entropy change of the $\mathrm{Gd}_{20} \mathrm{Dy}_{20} \mathrm{Er}_{20} \mathrm{Ho}_{20} \mathrm{~Tb}_{20} \mathrm{HEA}$ is about one order of magnitude larger than that of the reported NiFeCoCrPdx HEA systems $\left(<0.9 \mathrm{~J} \mathrm{~kg}^{-1} \mathrm{~K}^{-1}\right)$, similar to that of pure $\mathrm{Gd}(9.8$ $\mathrm{J} \mathrm{kg}^{-1} \mathrm{~K}^{-1}$ ). Second, MCE materials with coupled magneto-structural transitions suffer from cracking and fatigue, which severely limits the service lifetime. The current RE-HEA possesses good overall mechanical properties; its yield strength is above 250 $\mathrm{MPa}$ and the plasticity is larger than 20\%, as shown in Fig.10. Combining with its characteristic of the second-order phase transition without crystallographic structure change, the current HEA is anticipated to have a high cracking and fatigue-resistance [6]. Last, the current investigation opens up a possibility to synthesize MCE materials using Mischmetals [12] which is a more or less undefined alloy of RE elements in various naturally occurring proportions. By doing so, the production cost of the rare-earth MCE alloys will be significantly reduced, thus enabling real magnetic refrigeration applications.

\subsection{Origins of the giant MCE}

For magnetic refrigerators, the largest theoretical magnetic entropy change can be calculated as $\Delta S_{M}^{\max }=\mathrm{R} \ln (2 \mathrm{~J}+1)$, where $\mathrm{R}$ is the gas constant and $\mathrm{J}$ is the total angular momentum [31]. Estimates show that when applying REs and their alloys in 
magnetic refrigerators being operated over a wide temperature range, the value of $\Delta$ $S_{M}$ does not exceed $3 \%-5 \%$ of the value of $\Delta S_{M}^{\max }$, even under the magnetic field as high as 6T [31]. In our currently developed RE-HEA, its total angular momentum $\mathbf{J}$ can be calculated by an average of all five elements with $J_{m i x}=\sum_{i} c_{i} J_{i}$ according to the report of Luznik et al [13]. Consequently, the experimental maximum magnetic entropy change value $\Delta S_{M}=8.6 \mathrm{~J} \mathrm{~kg}^{-1} \mathrm{~K}^{-1}$ is $6.3 \%$ of the expected theoretical limit $\Delta$ $S_{M}^{\max }=\mathrm{R} \ln \left(2 J_{m i x}+1\right)\left(136.98 \mathrm{~J} \mathrm{~kg}^{-1} \mathrm{~K}^{-1}\right)$, which is larger than that in conventional magnetic refrigerators, also implying that complex coupling effects and magnetic structure may contribute to the enhanced MCE in the RE-HEA.

To better understand origins of the MCE and magnetic behavior in the RE-HEA, we investigated if the magnetic properties obey Vegard's rule of mixture, i.e., whether a given magnetic property of the "mixture" $Y_{m i x}$ is a compositional average of the properties $Y_{i}$ of constituent elements, $Y_{m i x}=\sum_{i} c_{i} Y_{i}$. We evaluate the departure of the experimental values $Y_{\exp }$ from the theoretical values $Y_{m i x}$ using the expression $\left(Y_{\text {exp }}-Y_{m i x}\right) / Y_{m i x}=\Delta \mathrm{Y} / Y_{m i x}$. Taking the literature-reported $T_{N}$ data of the constituent elements (see Table 1), we obtain $\Delta T_{N} / T_{N}^{m i x}=19 \%$. This observation implies that in addition to compositional average of magnetic properties, there may exist complex coupling effects from the disordering structure of multiple elements.

As elaborated earlier, the $\mathrm{Gd}_{20} \mathrm{Dy}_{20} \mathrm{Er}_{20} \mathrm{Ho}_{20} \mathrm{~Tb}_{20}$ HEA can be taken as random mixing of the five chemical elements on the single HCP lattice. The magnetic state can be determined predominantly by the random local pinning potential that impedes 
thermally assisted spin reorientations so that in zero external field the spins are in random directions, as schematically shown in Fig.11a. Under an external magnetic field, the spins tend to orientate and magnetic entropy changes. Due to each constituent element having different magnetic moment, the element with a smaller magnetic moment will firstly orientate (Fig. 11b), whilst orientation of the element with a larger magnetic moment needs a larger external magnetic force (Fig. 11c), which is different from the conventional magnets with a specified magnetic moment. Therefore, due to the random chemical distributions of the five elements, complex magnetic structure and exchange coupling of the multiple principle elements exist in the RE-HEA, which prolonged the total magnetic phase transition process, thus leading to a sluggish magnetic transition in a wide temperature range and eventually resulting in the large $\mathrm{RC}$ and magnetocaloric effects. 


\section{Conclusions}

Phase formation of multiple principle-element rare-earth alloys was investigated and single phase structure was only found in the quinary alloys with high entropy of mixing of $1.61 \mathrm{R}$, suggesting that it is entropy, rather than other factors such as enthalpy, atomic size and electronegativity, that dominates phase formation in the current rare-earth system. The quinary RE-HEA exhibited large MCE $\left(8.6 \mathrm{~J} \mathrm{~kg}^{-1} \quad \mathrm{~K}^{-1}\right.$ at the $5 \mathrm{~T}$ magnetic field) and the largest $\mathrm{RC}$ reported so far (about $627 \mathrm{~J} \mathrm{~kg}^{-1}$ at the $5 \mathrm{~T}$ magnetic field). This RE-HEA exhibited a second order magnetic phase transition at its Neel temperature, a small hysteresis loss and ductile mechanical behavior. The combination of all unique properties makes the current RE-HEA promising for magnetic refrigeration applications. Our analysis indicates that strong chemical disorder resulted from the high configuration entropy makes magnetic ordering difficult, thus giving rise to a sluggish magnetic phase transition and enhanced magnetocaloric effect. Although theoretical description of the magnetocaloric properties of the HEA is still a challenging problem because of their random local distortion of the lattice and random distribution of five or more chemical elements on the otherwise simple average lattice, there is no doubt that the chemical disorder and sluggish kinetics resulting from the high configuration entropy make HEAs a novel class of magnetocaloric materials. 


\section{Acknowledgements}

This research was supported by National Natural Science Foundation of China (Nos. 51531001, 51671018, 51422101, 51371003 and51271212), 111 Project (B07003), International S\&T Cooperation Program of China (2015DFG52600) and Program for Changjiang Scholars and Innovative Research Team in University (IRT_14R05). YW acknowledges the financial support from the Top-Notch Young Talents Program and Fundamental Research Fund for the Central Universities (Nos. FRF-TP-15-004C1). The work of Tong was sponsored by the Scientific User Facilities Division, Office of Basic Energy Sciences, United States Department of Energy. Oak Ridge National Laboratory is managed by UT-Battelle, LLC, for the US Department of Energy (DOE) under contract no. DE-AC05- 00OR22725. 


\section{References}

[1]. E. Warburg, Magnetische untersuchungen. Ann. Phys. 249 (1881) 141-164.

[2]. W.F. Giauque, D.P. MacDougall, Attainment of Temperatures Below $1^{\circ}$ Absolute by Demagnetization of $\mathrm{Gd}_{2}\left(\mathrm{SO}_{4}\right)_{38} \mathrm{H}_{2} \mathrm{O}$. Phys. Rev. 43 (1933) 768.

[3]. A.F. Lacaze, R. Beranger, G.B. Mardion, G. Claudet, A.A. Lacaze, Double acting reciprocating magnetic refrigerator: recent improvements, in Advances in Cryogenic Engineering. (Springer, 1984) 573-579.

[4]. R.D. Shull, R.D. McMichael, J.J. Ritter, Magnetic nanocomposites for magnetic refrigeration. Nanostruct. mater. 2 (1993) 205-211.

[5]. G.V. Brown, Magnetic heat pumping near room temperature. J. Appl. Phys. 47 (1976) 3673-3680.

[6]. D.D. Belyea, M.S. Lucas, E. Michel, J. Horwath, C.W. Miller, Tunable magnetocaloric effect in transition metal alloys. Sci. Rep. 5 (2015) 351-360.

[7]. F. Hu, B. Shen, J. Sun, Magnetic entropy change in $\mathrm{Ni}_{51 .} \mathrm{Mn}_{22.7} \mathrm{Ga}_{25.8}$ alloy. Appl. Phys. Lett. 76 (2000) 3460-3462.

[8]. J.W. Yeh, S. K. Chen, S. J. Lin, J. Y. Gan, T. S. Chin, T. T. Shun, C. H. Tsau, S. Y. Chang, Nanostructured High - Entropy Alloys with Multiple Principal Elements: Novel Alloy Design Concepts and Outcomes. Adv. Eng. Mater. 6 (2004) 299-303.

[9]. J.W. Yeh, Recent progress in high-entropy alloys. Eur. J. Control. 31 (2006) 633-648.

[10]. Y. Zhang, T. T. Zuo, Z. Tang, M. C. Gao, K. A. Dahmen, P. K. Liaw, Z. P. Lu, Microstructures and properties of high-entropy alloys. Prog. Mater. Sci. 61 (2014) 1-93.

[11]. B. Cantor, I.T.H. Chang, P. Knight, A.J.B. Vincent, Microstructural development in equiatomic multicomponent alloys. Mater. Sci. Eng. A. 375 (2004) 213-218.

[12]. M. Feuerbacher, M. Heidelmann, C. Thomas, Hexagonal high-entropy alloys. Mater. Res. Lett. 3 (2015) 1-6.

[13]. J. Lužnik, P. Koželj, S. Vrtnik, A. Jelen, Z. Jagličić, A. Meden, M. Feuerbacher, J. Dolinšek, Complex magnetism of Ho-Dy-Y-Gd-Tb hexagonal high-entropy alloy. Phys. Rev. B. 92 (2015) 224201.

[14]. M.H. Yu, L.H. Lewis, A.R. Moodenbaugh, Large magnetic entropy change in the metallic antiperovskite Mn3GaC. J. Appl. Phys. 93 (2003) 10128-10130.

[15]. B.S. Wang, P. Tong, Y. P. Sun, X. Luo, X. B. Zhu, G. Li, X. D. Zhu, S. B. Zhang, Z. R. Yang, W. H. Song, Large magnetic entropy change near room temperature in antiperovskite SnCMn3. Europhys. Lett. 85 (2009) 3699-3704.

[16]. T. Hashimoto, T. Numasawa, M. Shino, T. Okada, Magnetic refrigeration in the temperature range from $10 \mathrm{~K}$ to room temperature: the ferromagnetic refrigerants. Cryogenics.21 (1981) 647-653.

[17]. M.E. Wood, W.H. Potter, General analysis of magnetic refrigeration and its optimization using a new concept: maximization of refrigerant capacity. Cryogenics, 25 (1985) 667-683.

[18]. A.M. Tishin, Y.I. Spichkin, The Magnetocaloric Effect and Its Applications. IOP. New York, 2003.

[19]. P. Gorria, J.L.S. Llamazares, P. Álvarez, M.J. Pérez, J.S. Marcos, J.A. Blanco, Relative cooling power enhancement in magneto-caloric nanostructured $\mathrm{Pr}_{2} \mathrm{Fe}_{17}$. J. Phys. D: Appl. Phys. 41 (2008) 3080-3090.

[20].X. Yang, Y. Zhang, Prediction of high-entropy stabilized solid-solution in multi-component alloys. Mater. Chem. Phys. 132 (2012) 233-238. 
[21]. Y. Zhang, Y.J. Zhou, J.P. Lin, G.L. Chen, P.K. Liaw, Solid - solution phase formation rules for multi - component alloys. Adv. Eng. Mater. 261 (2003) 7-11.

[22]. T. Egami, Y. Waseda, Atomic size effect on the formability of metallic glasses. J. Non-Cryst. Solids 64 (1984) 113-134.

[23]. Y.F. Ye, C.T. Liu, Y. Yang, A geometric model for intrinsic residual strain and phase stability in high entropy alloys. Acta Mater. 94 (2015) 152-161.

[24]. S. Fang, X. Xiao, X. Lei, W. Li, Y. Dong, Relationship between the widths of supercooled liquid regions and bond parameters of Mg-based bulk metallic glasses. J. Non-Cryst. Solids 321 (2003) 120-125.

[25]. S. Guo, C. Ng, J. Lu, C. T. Liu, Effect of valence electron concentration on stability of fcc or bcc phase in high entropy alloys. J. Appl. Pys.109 (2011) 645-647.

[26]. S. Guo, C.T. Liu, Phase stability in high entropy alloys: formation of solid-solution phase or amorphous phase. Prog. Nat. Sci. 21 (2011) 433-446.

[27].B.K. Banerjee, On a generalised approach to first and second order magnetic transitions. Phys. Let. 12 (1964) 16-17.

[28]. A. Tari, The specific heat of matter at low temperatures. World Scientific. 348 (2003).

[29]. V.K. Pecharsky, K.A. Gschneidner, Giant magnetocaloric effect in Gd 5 (Si 2 Ge 2). Phys. Rev. Lett. 78 (1997) 4494-4497.

[30]. V. Provenzano, A.J. Shapiro, R.D. Shull, Reduction of hysteresis losses in the magnetic refrigerant Gd5Ge2Si2 by the addition of iron. Nature. 429 (2004) 853-857.

[31]. A.M. Tishin, Magnetic refrigeration in the low - temperature range. J. Appl. Phys. 68 (1990) 6480-6484.

[32]. K.A. Gschneidner, Physical properties of the rare earth metals. J. Phase Equilib, 11 (1990) 216-224.

[33]. L. Liang, X. Hui, Y. Wu, G. Chen, Large magnetocaloric effect in $\operatorname{Gd}_{36} \mathrm{Y}_{20} \mathrm{Al}_{24} \mathrm{Co}_{20}$ bulk metallic glass. J. Alloy Compd. 457 (2008) 541-544.

[34]. T.D. Shen, R.B. Schwarz, J.Y. Coulter, J.D. Thompson, Magnetocaloric effect in bulk amorphous $\mathrm{Pd}_{40} \mathrm{Ni}_{22.5} \mathrm{Fe}_{17.5} \mathrm{P}_{20}$ alloy. J. Appl. Phys. 91 (2002) 5240-5245.

[35]. J. Du, Q. Zheng, E. Brück, K.H.J. Buschow, W.B. Cui, W. J. Feng, Z.D. Zhang, Spin-glass behavior and magnetocaloric effect in Tb-based bulk metallic glass. J. Magn. Magn. Mater. 321 (2009) 413-417.

[36]. L. Liang, X. Hui, C.M. Zhang, G.L. Chen, A Dy-based bulk metallic glass with high thermal stability and excellent magnetocaloric properties. J. Alloy Compd. 463 (2008) 30-33.

[37]. V. Franco, J.S. Blazquez, A. Conde, The influence of Co addition on the magnetocaloric effect of Nanoperm-type amorphous alloys. J. Appl. Phys. 100 (2006) 064307.

[38]. V. Franco, J.M. Borrego, C.F. Conde, A. Conde, Refrigerant capacity of FeCrMoCuGaPCB amorphous alloys. J. Appl. Phys. 100 (2006) 083903. 


\section{Table and Figure Captions}

Table 1. Lattice parameters $a$ and $c$, atomic radius $\gamma$, Curie temperature $T_{c}$, Neel temperature $T_{N}$ and total angular momentum $\mathbf{J}$ [32] of constituent RE elements and the investigated $\mathrm{Gd}_{20} \mathrm{Dy}_{20} \mathrm{Er}_{20} \mathrm{Ho}_{20} \mathrm{~Tb}_{20} \mathrm{HEA}$. The theoretical values (HEA theor.) were calculated based on the "rule of mixtures".

Table 2. Structure types, magnetic entropy changes $\Delta \mathrm{S}_{\mathrm{M}}$, refrigerant capacity $\mathrm{RC}$, and magnetic phase transition temperature $T_{c} / T_{N}$ of the equimolar rare-earth alloys in comparison with those of the conventional magnetocaloric materials.

Fig.1 (a) XRD patterns of the $\mathrm{Gd}_{20} \mathrm{Dy}_{20} \mathrm{Er}_{20} \mathrm{Ho}_{20} \mathrm{~Tb}_{20}, \mathrm{Gd}_{25} \mathrm{Er}_{25} \mathrm{Ho}_{25} \mathrm{~Tb}_{25}$, $\mathrm{Dy}_{25} \mathrm{Er}_{25} \mathrm{Ho}_{25} \mathrm{~Tb}_{25}$ and $\mathrm{Er}_{33.33} \mathrm{Ho}_{33.33} \mathrm{~Tb}_{33.34}$, (b) SEM image of the $\mathrm{Gd}_{20} \mathrm{Dy}_{20} \mathrm{Er}_{20} \mathrm{Ho}_{20} \mathrm{~Tb}_{20}$ alloy, (c) XRD patterns of the $\mathrm{Gd}_{20} \mathrm{Dy}_{20} \mathrm{Er}_{20} \mathrm{Ho}_{20} \mathrm{~Tb}_{20} \mathrm{HEA}$ from $20 \mathrm{~K}$ to room temperature.

Fig.2 (a) A typical STEM image of the $\mathrm{Gd}_{20} \mathrm{Dy}_{20} \mathrm{Er}_{20} \mathrm{Ho}_{20} \mathrm{~Tb}_{20}$ HEA. SAED patterns along different zone axes of the hexagonal structure: (b)[2110], (c)[0110], and (d)[0001] zone axis.

Fig.3 Temperature dependences of zero-field-cooling (ZFC) magnetizations of the $\mathrm{Gd}_{20} \mathrm{Dy}_{20} \mathrm{Er}_{20} \mathrm{Ho}_{20} \mathrm{~Tb}_{20}$ HEA under various magnetic field in the temperature range between 50 and $300 \mathrm{~K}$. The inset shows the enlarged peak of the M-T curve of $\mathrm{Gd}_{20} \mathrm{Dy}_{20} \mathrm{Er}_{20} \mathrm{Ho}_{20} \mathrm{~Tb}_{20}$ HEA measured under a magnetic field of $0.3 \mathrm{~T}$ from 170 to 200K, along with the corresponding Gaussion fit.

Fig.4 (a) Initial isothermal magnetization $\mathrm{M}(\mathrm{H})$ curves of the $\mathrm{Gd}_{20} \mathrm{Dy}_{20} \mathrm{Er}_{20} \mathrm{Ho}_{20} \mathrm{~Tb}_{20}$ HEA under the magnetic field of $5 \mathrm{~T}$ in the temperature range from 90 to $230 \mathrm{~K}$ with an interval of 5K. (b) Magnetic hysteresis in $\mathrm{M}(\mathrm{H})$ at 90, 115, 140, 160, 185 and 230 $\mathrm{K}$ from $0-2 \mathrm{~T}$. 
Fig.5 Magnetic entropy change $\left|\Delta S_{M}\right|$ of the $\mathrm{Gd}_{20} \mathrm{Dy}_{20} \mathrm{Er}_{20} \mathrm{Ho}_{20} \mathrm{~Tb}_{20}$ HEA as a function of temperature under a maximum applied field of 1, 2, 3, 4 and $5 \mathrm{~T}$. The shaded region in the inset shows numerically integrated area for calculating the RC-2 value; $T_{\text {hot }}(107 \mathrm{~K})$ and $T_{\text {cold }}(211 \mathrm{~K})$ refer to the limits of integration for calculating the shaded area.

Fig.6 Refrigerant capacities RC-1, RC-2, and RC-3 as a function of magnetic field $\mathrm{H}$ for the $\mathrm{Gd}_{20} \mathrm{Dy}_{20} \mathrm{Er}_{20} \mathrm{Ho}_{20} \mathrm{~Tb}_{20} \mathrm{HEA}$.

Fig.7 Arrott plots (i.e., $\mathrm{M}^{2}$ vs $\mathrm{H} / \mathrm{M}$ ) at different testing temperatures. The inset shows the Arrott curve at several specific temperatures for a clearer illustration.

Fig.8 Zero-field specific heat $\mathrm{C}_{\mathrm{p}}$ of the $\mathrm{Gd}_{20} \mathrm{Dy}_{20} \mathrm{Er}_{20} \mathrm{Ho}_{20} \mathrm{~Tb}_{20}$ HEA in the temperature range between 65 and $275 \mathrm{~K}$, clearly showing a $\lambda$-like peak at its Neel temperature $T_{N}=186 \mathrm{~K}$.

Fig.9 Hysteresis loss of the $\mathrm{Gd}_{20} \mathrm{Dy}_{20} \mathrm{Er}_{20} \mathrm{Ho}_{20} \mathrm{~Tb}_{20}$ HEA plotted as a function of temperature.

Fig.10 Compressive stress-strain curves of the as-cast $\mathrm{Gd}_{20} \mathrm{Dy}_{20} \mathrm{Er}_{20} \mathrm{Ho}_{20} \mathrm{~Tb}_{20}$ alloy. The HEA shows a good combination of mechanical properties; the yield strength is above $250 \mathrm{MPa}$ and the plasticity is larger than $20 \%$.

Fig.11 Schematic illustration of spins ordering with increasing magnetic field within one hexagonal layer of the HCP lattice of the $\mathrm{Gd}_{20} \mathrm{Dy}_{20} \mathrm{Er}_{20} \mathrm{Ho}_{20} \mathrm{~Tb}_{20}$ HEA. The Gd, Dy, Er, Ho, and $\mathrm{Tb}$ ions are respectively represented by circles with different sizes. 
The magnetic moments of Gd, Dy, Er, Ho, and Tb ions are drawn by arrows, and the length of the arrow is proportional to the size of the moment. Apparently, the spins were in random directions in the zero external field, and they tend to orientate under an increasing external magnetic field. 
Fig.1

(a)

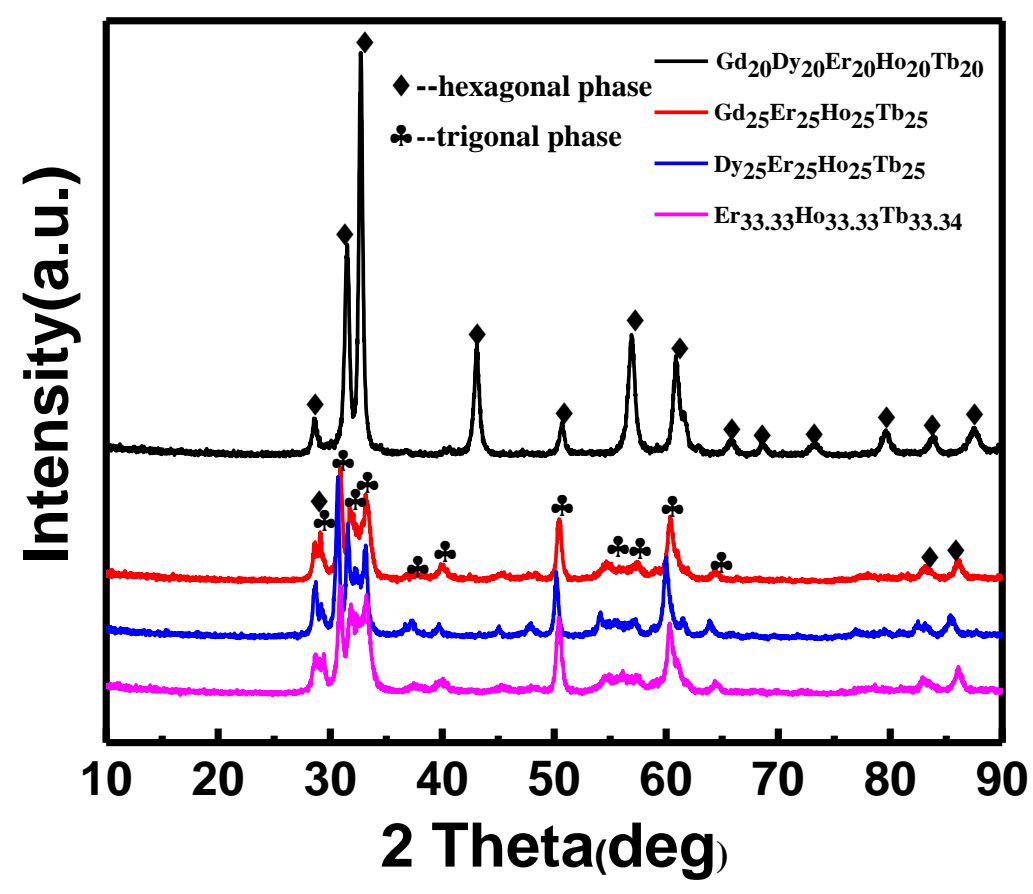

(b)

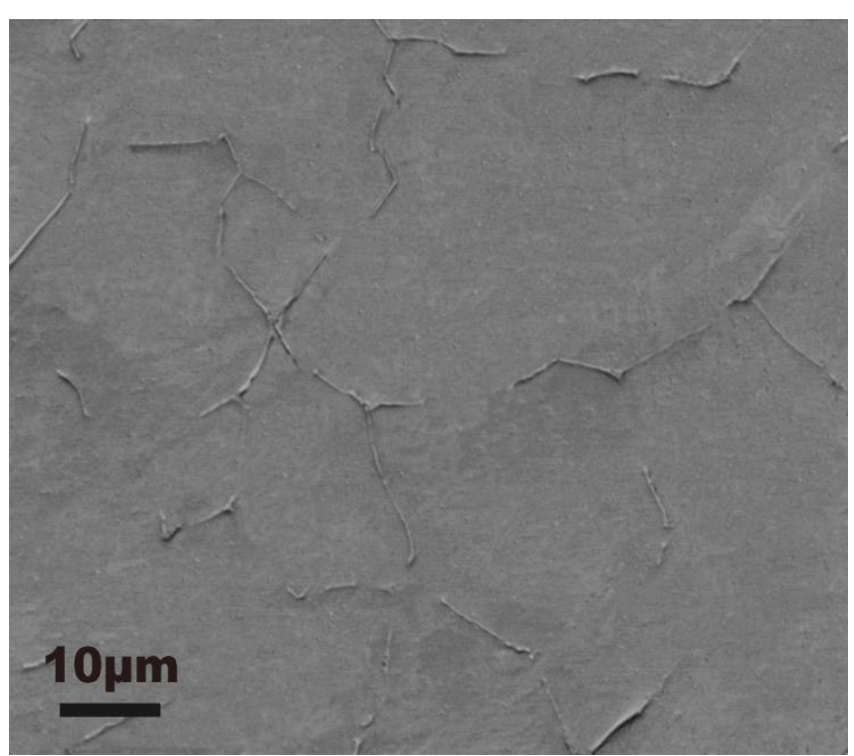


(c)

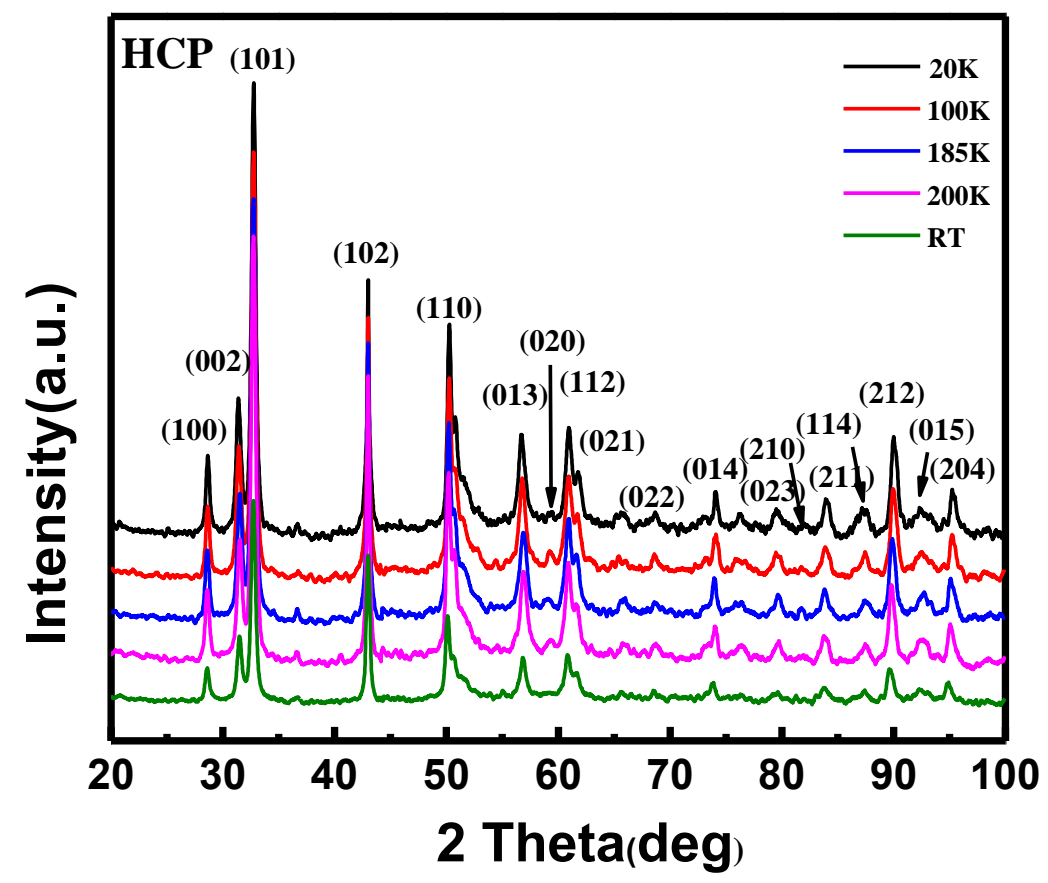



Fig.2

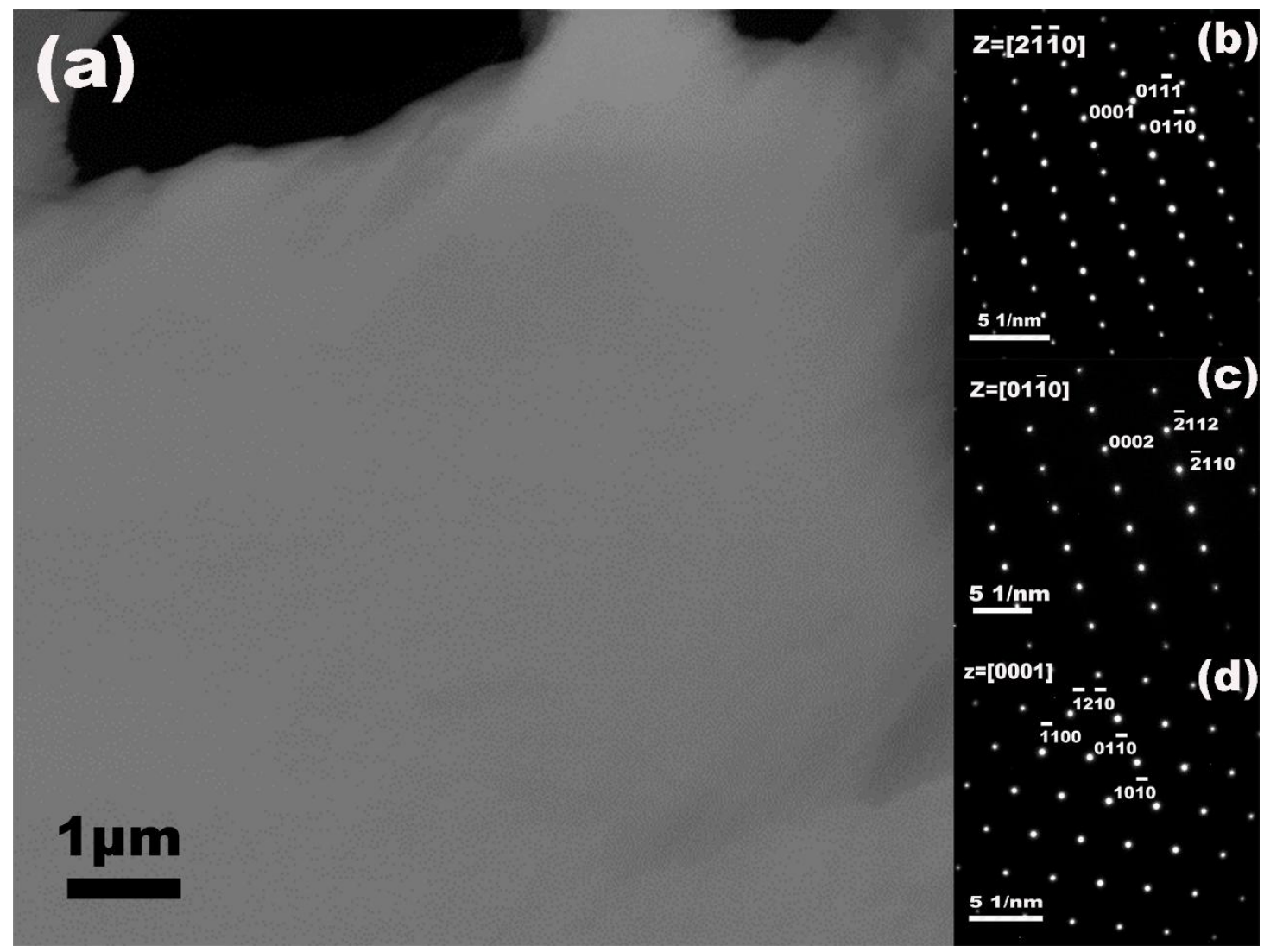


Fig.3

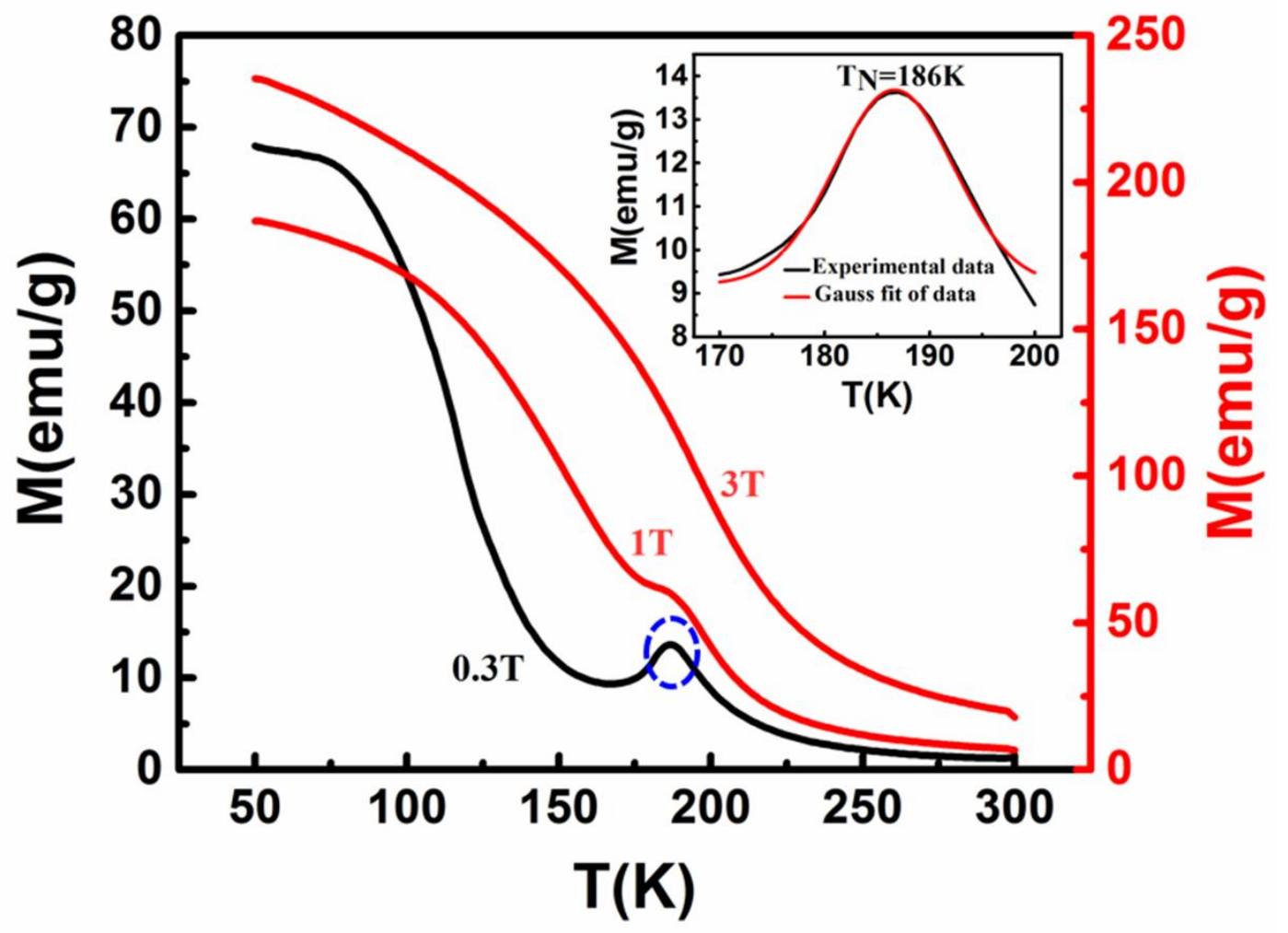


Fig.4
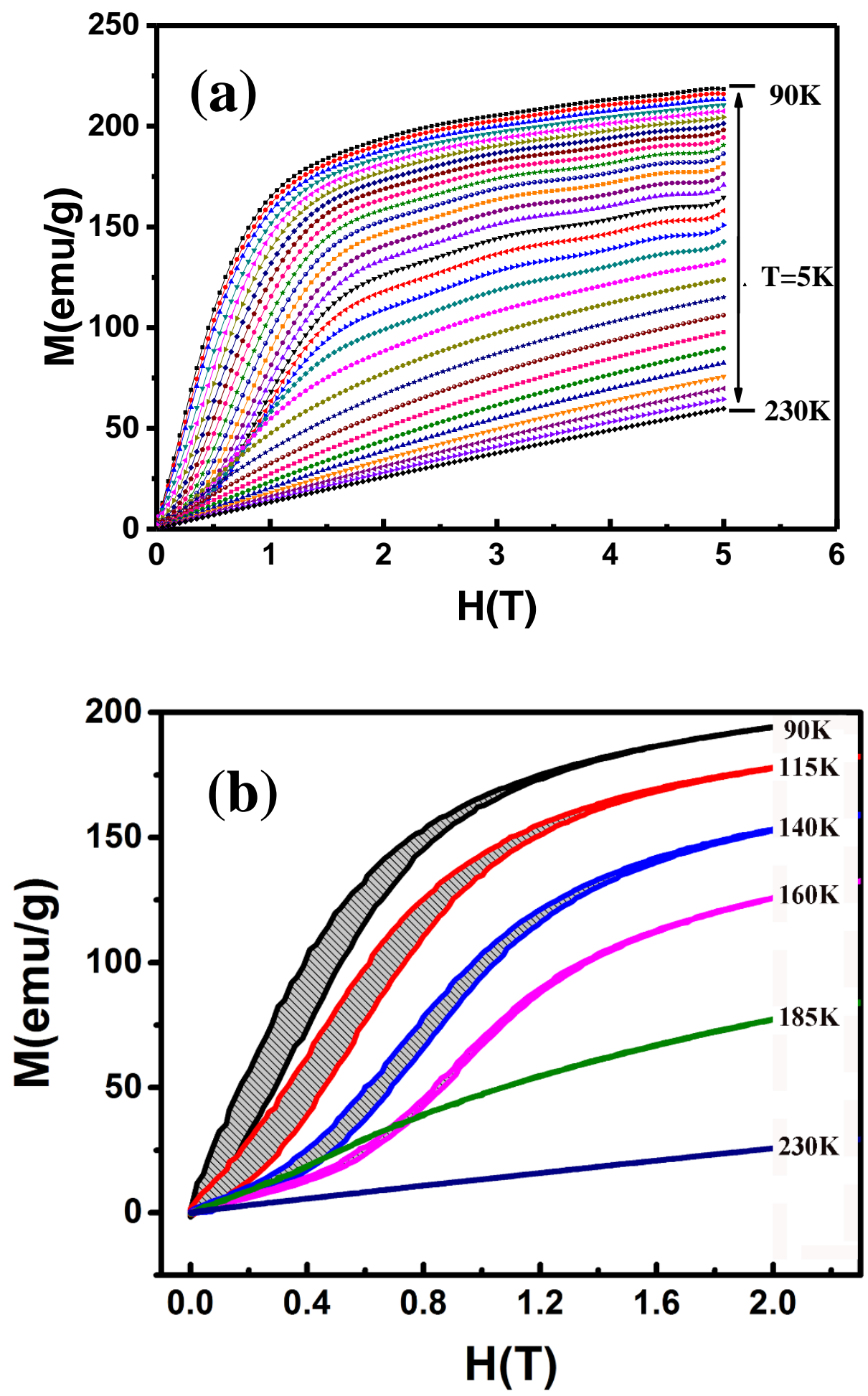
Fig.5

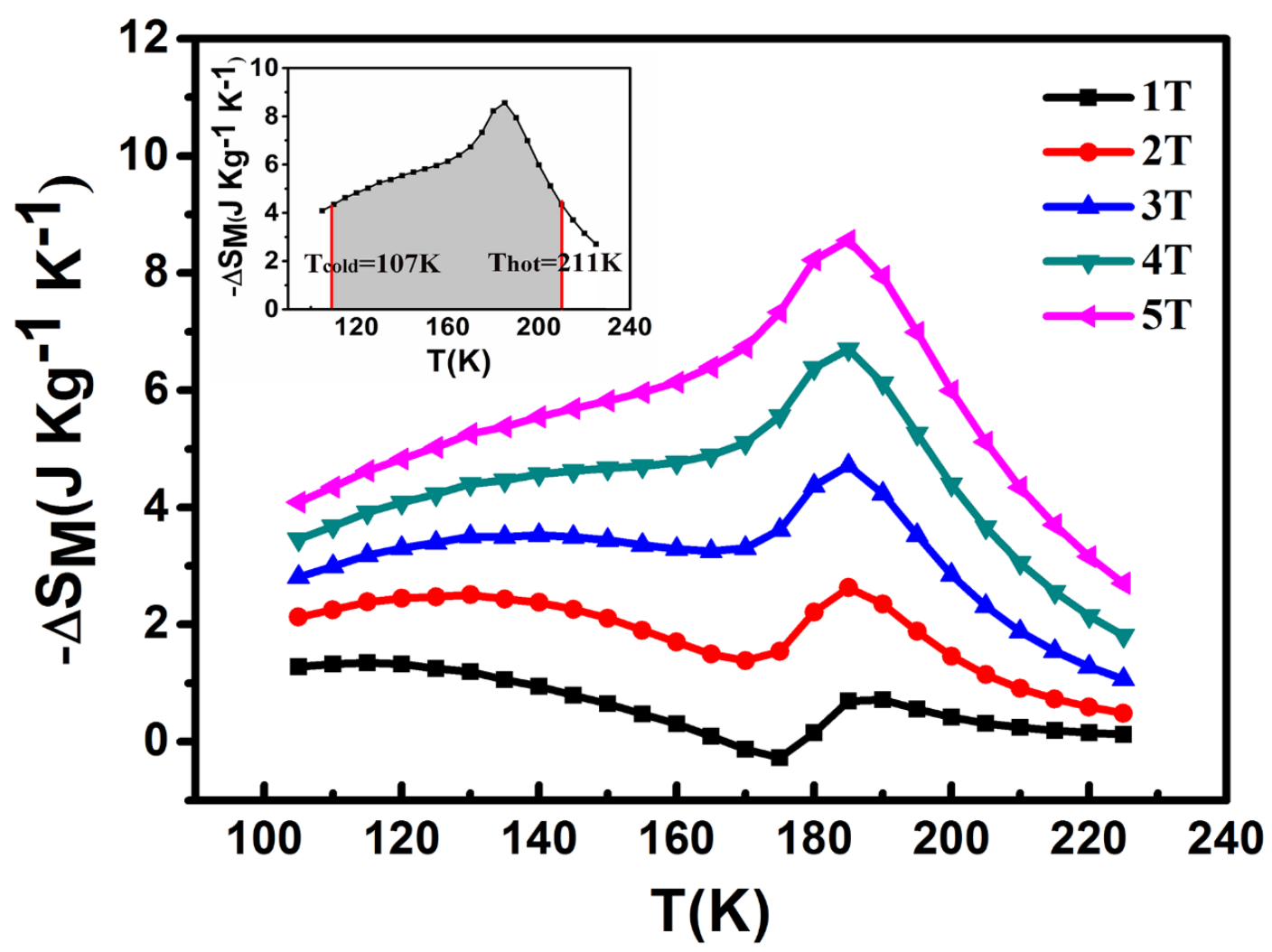


Fig.6

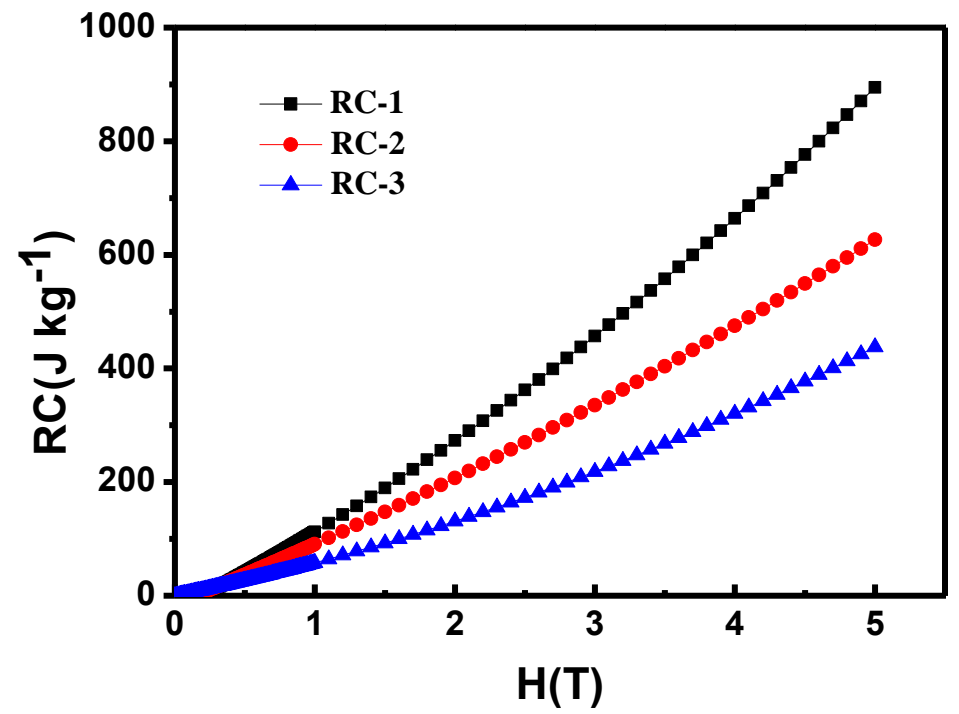


Fig.7

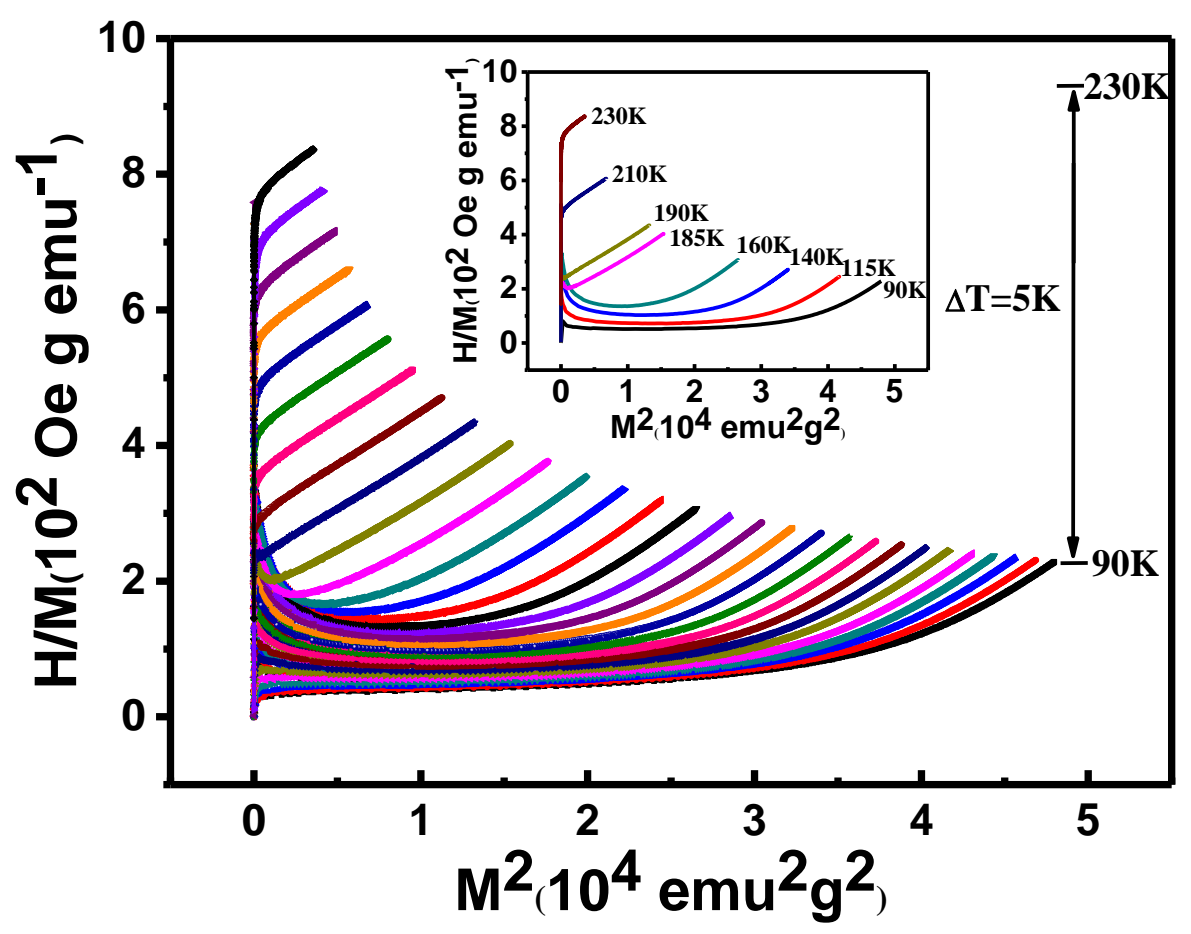


Fig. 8

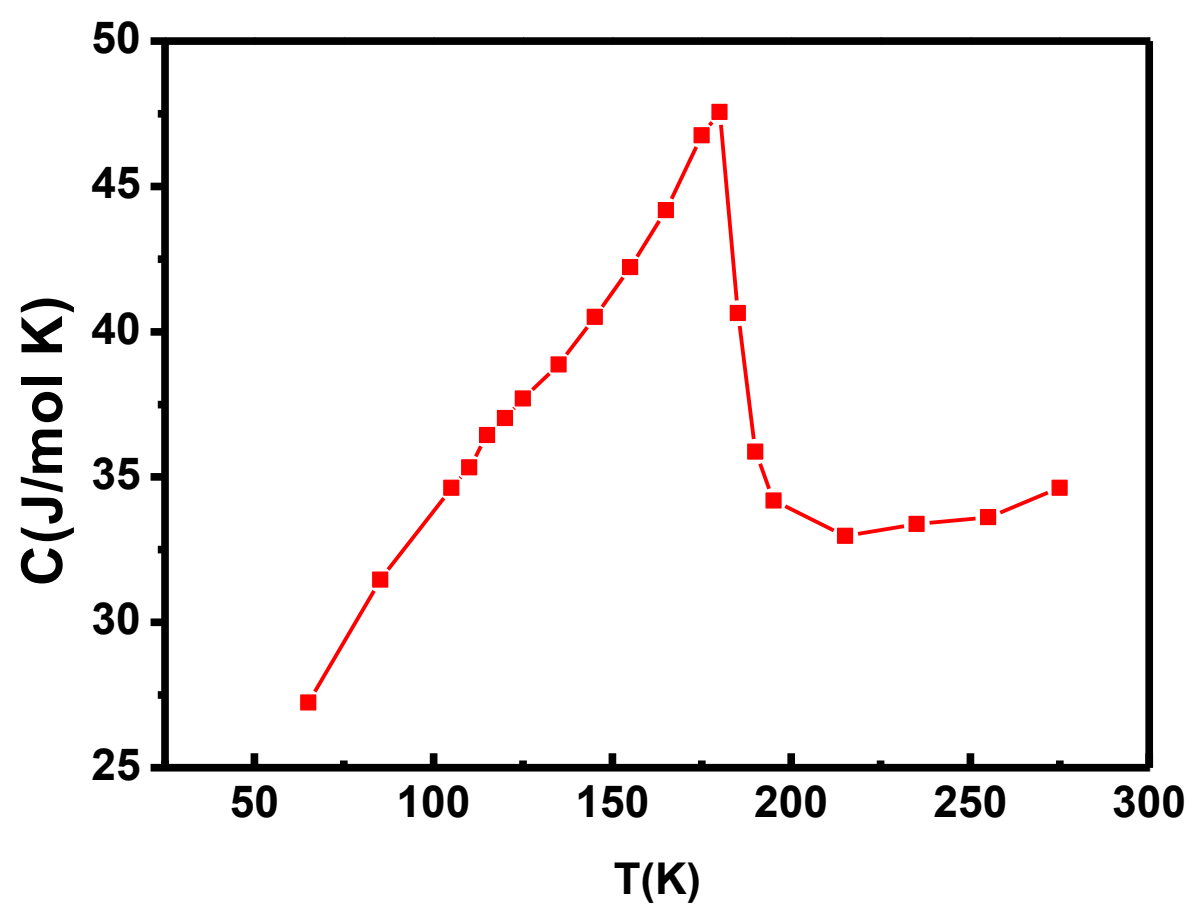


Fig.9

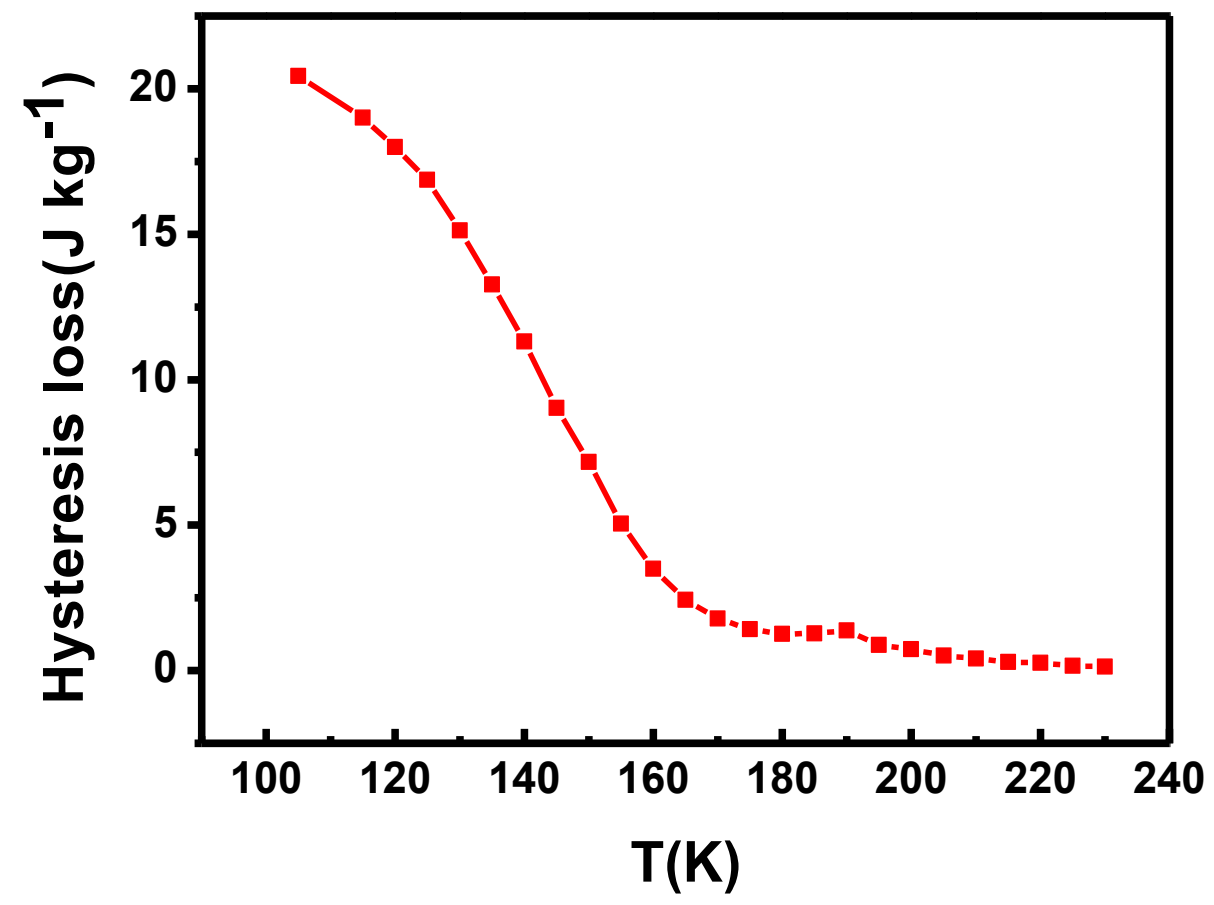


Fig.10

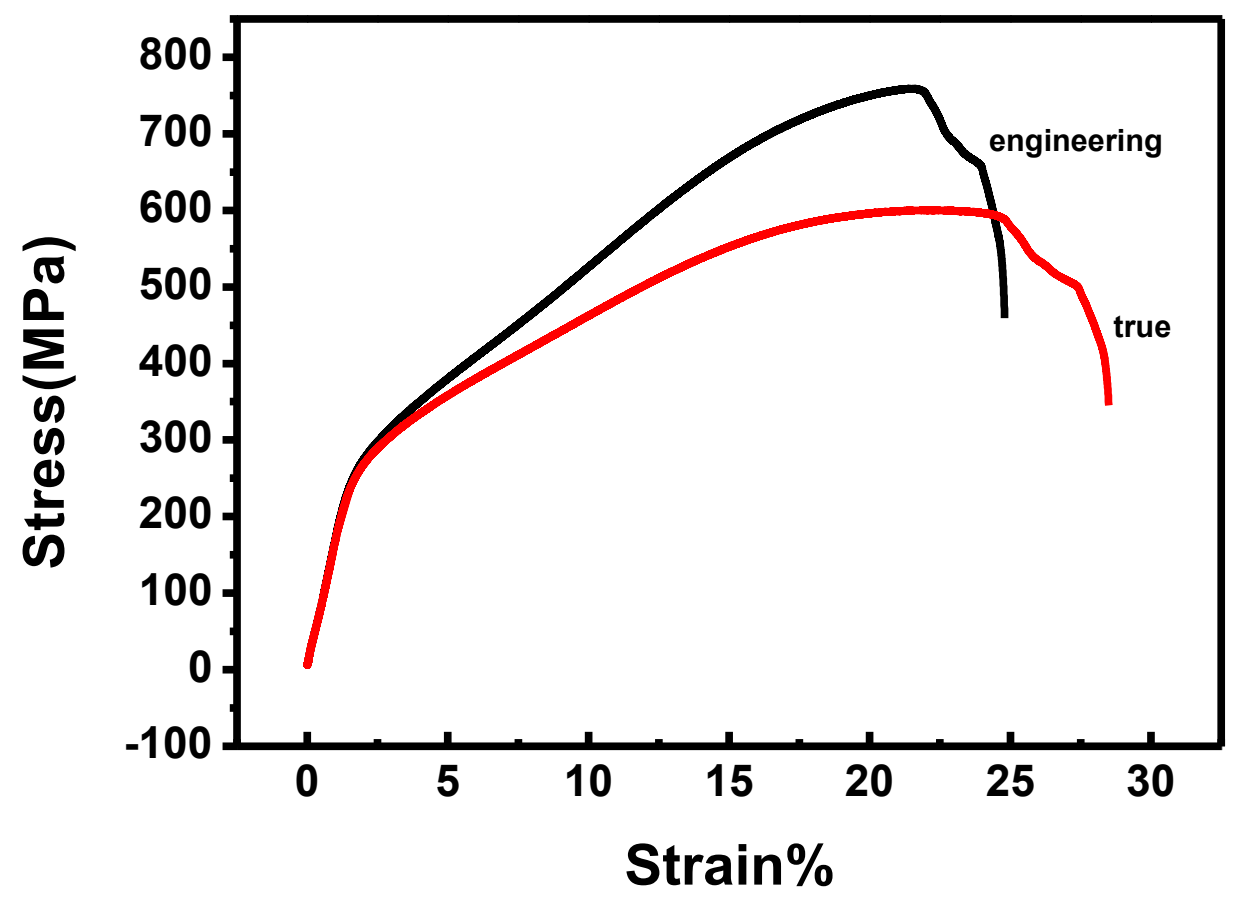


Fig.11
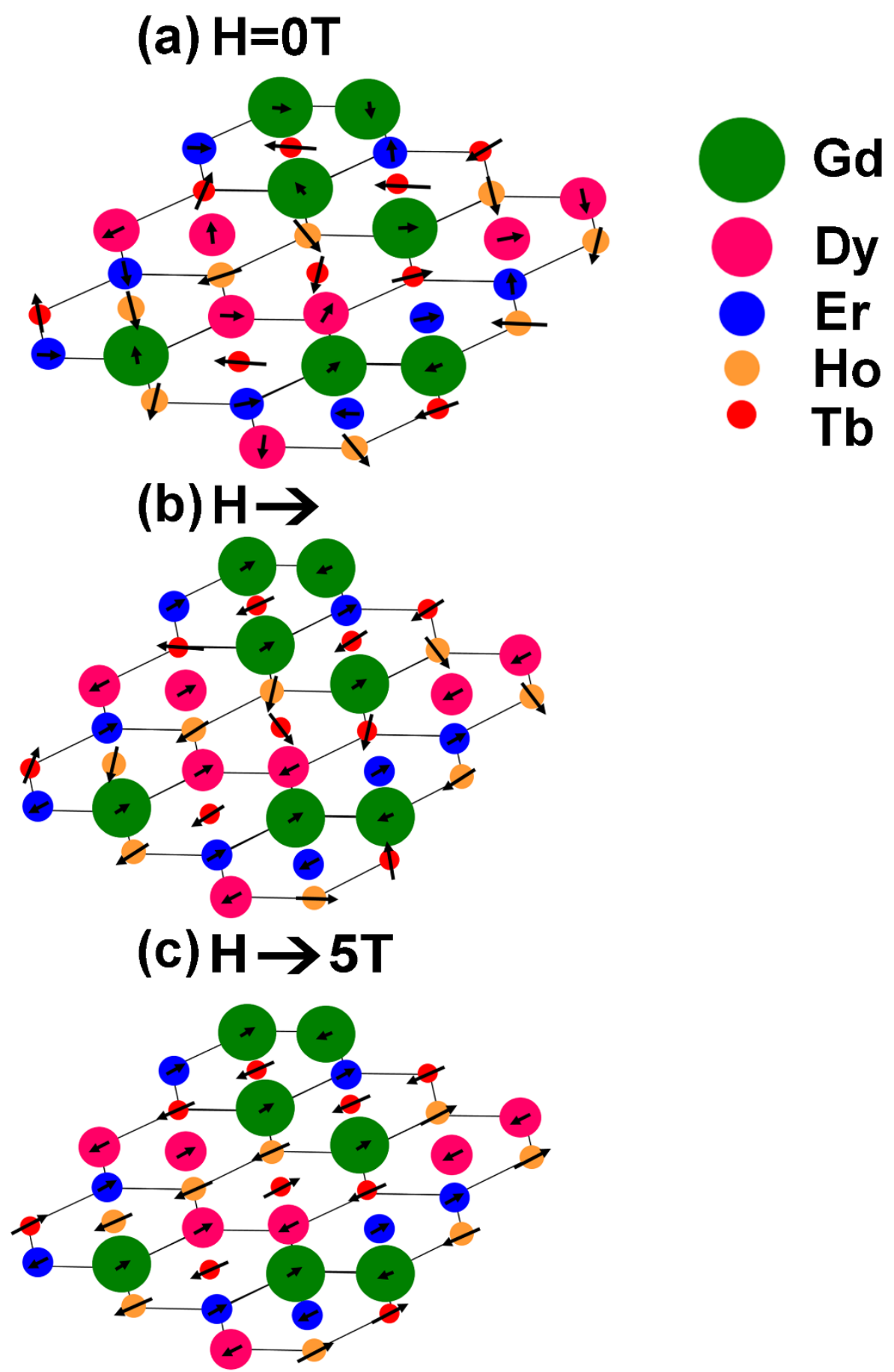
Table 1

\begin{tabular}{|c|c|c|c|c|c|c|c|}
\hline & Gd & Dy & $\mathbf{E r}$ & Ho & $\mathbf{T b}$ & $\begin{array}{c}\text { HEA } \\
\text { theor. }\end{array}$ & $\begin{array}{c}\text { HEA } \\
\text { exp. }\end{array}$ \\
\hline Struture & HCP & HCP & HCP & HCP & HCP & HCP & HCP \\
\hline$a\left(\begin{array}{l}0 \\
A\end{array}\right)$ & 3.634 & 3.592 & 3.559 & 3.578 & 3.606 & 3.594 & 3.599 \\
\hline$c\left(\begin{array}{l}0 \\
A\end{array}\right)$ & 5.781 & 5.650 & 5.585 & 5.618 & 5.697 & 5.666 & 5.678 \\
\hline$\gamma\left(\begin{array}{l}\circ \\
A\end{array}\right)$ & 1.8013 & 1.774 & 1.7566 & 1.7661 & 1.7833 & 1.7763 & \\
\hline$T_{N}(\mathbf{K})$ & & 179 & 85 & 132 & 230 & 156.5 & 186 \\
\hline $\mathbf{J}$ & $7 / 2$ & $15 / 2$ & $15 / 2$ & 8 & 6 & $13 / 2$ & \\
\hline
\end{tabular}


Table 2

\begin{tabular}{|c|c|c|c|c|c|c|c|}
\hline Material & Structure & $\mathbf{T}_{\mathbf{N}}(\mathbf{K})$ & $\mathbf{T}_{\mathbf{c}}(\mathbf{K})$ & $\begin{array}{l}\text { Applied } \\
\text { field(T) }\end{array}$ & $\begin{array}{c}\Delta \mathbf{S}_{\mathrm{M}} \\
\left(\mathrm{J} \mathrm{kg}^{-1} \mathbf{K}^{-1}\right)\end{array}$ & $\begin{array}{c}\mathbf{R C} \\
\left(\mathrm{J} \mathrm{kg}^{-1}\right)\end{array}$ & Reference \\
\hline $\mathrm{Gd}_{20} \mathrm{Dy}_{20} \mathrm{Er}_{20} \mathrm{Ho}_{20} \mathrm{~Tb}_{20}$ & HCP & 186 & & 5 & 8.6 & 627 & This work \\
\hline $\mathrm{Gd}_{25} \mathrm{Er}_{25} \mathrm{Ho}_{25} \mathrm{~Tb}_{25}$ & HCP+TRI & 139 & & 3 & 4.8 & 137 & This work \\
\hline $\mathrm{Dy}_{25} \mathrm{Er}_{25} \mathrm{Ho}_{25} \mathrm{~Tb}_{25}$ & HCP+TRI & 52 & & 3 & 0.65 & 27.3 & This work \\
\hline $\mathrm{Er}_{33.33} \mathrm{Ho}_{33.33} \mathbf{T b}_{33.34}$ & HCP+TRI & 88 & & 3 & 3 & 150 & This work \\
\hline NiFeCoCrPdx & FCC & & 225 & 5 & $<0.9$ & $<175$ & [6] \\
\hline $\mathbf{G d}_{36} \mathbf{Y}_{20} \mathrm{Al}_{24} \mathrm{Co}_{20}$ & BMGs & & 53 & 5 & 7.76 & 459 & [33] \\
\hline $\mathbf{P d}_{40} \mathrm{Ni}_{22.5} \mathrm{Fe}_{17.5} \mathbf{P}_{20}$ & BMGs & & 94 & 5 & 0.58 & - & {$[34]$} \\
\hline $\mathrm{Tb}_{55} \mathrm{Co}_{20} \mathrm{Al}_{25}$ & HE-BMGs & & 105 & 5 & 7.5 & 352 & {$[35]$} \\
\hline $\mathrm{Dy}_{36} \mathrm{Ho}_{20} \mathrm{Co}_{20} \mathrm{Al}_{24}$ & HE-BMGs & & 23 & 5 & 9.49 & 417 & {$[36]$} \\
\hline $\mathrm{Fe}_{78} \mathrm{Co}_{5} \mathrm{Zr}_{6} \mathrm{~B}_{10} \mathrm{Cu}_{1}$ & Glass ribbon & & 480 & 1.5 & 1.6 & 82 & [37] \\
\hline $\mathrm{Fe}_{65.5} \mathrm{Cr}_{4} \mathrm{Mo}_{3} \mathrm{Cu}_{1} \mathrm{Ga}_{4} \mathrm{P}_{12} \mathrm{C}_{5} \mathrm{~B}_{5.5}$ & Glass ribbon & & 500 & 1.5 & 1.08 & 59 & [38] \\
\hline Gd & HCP & & 293 & 5 & 9.8 & - & [5] \\
\hline $\mathbf{G d}_{5} \mathbf{S i}_{2} \mathbf{G e}_{2}$ & monoclinic & & 276 & 5 & 18.6 & 306 & [29] \\
\hline $\mathbf{G d}_{5} \mathrm{Si}_{2} \mathbf{G e}_{1.9} \mathbf{F e}_{0.1}$ & multiple phase & & 320 & 5 & 7 & 360 & [30] \\
\hline
\end{tabular}




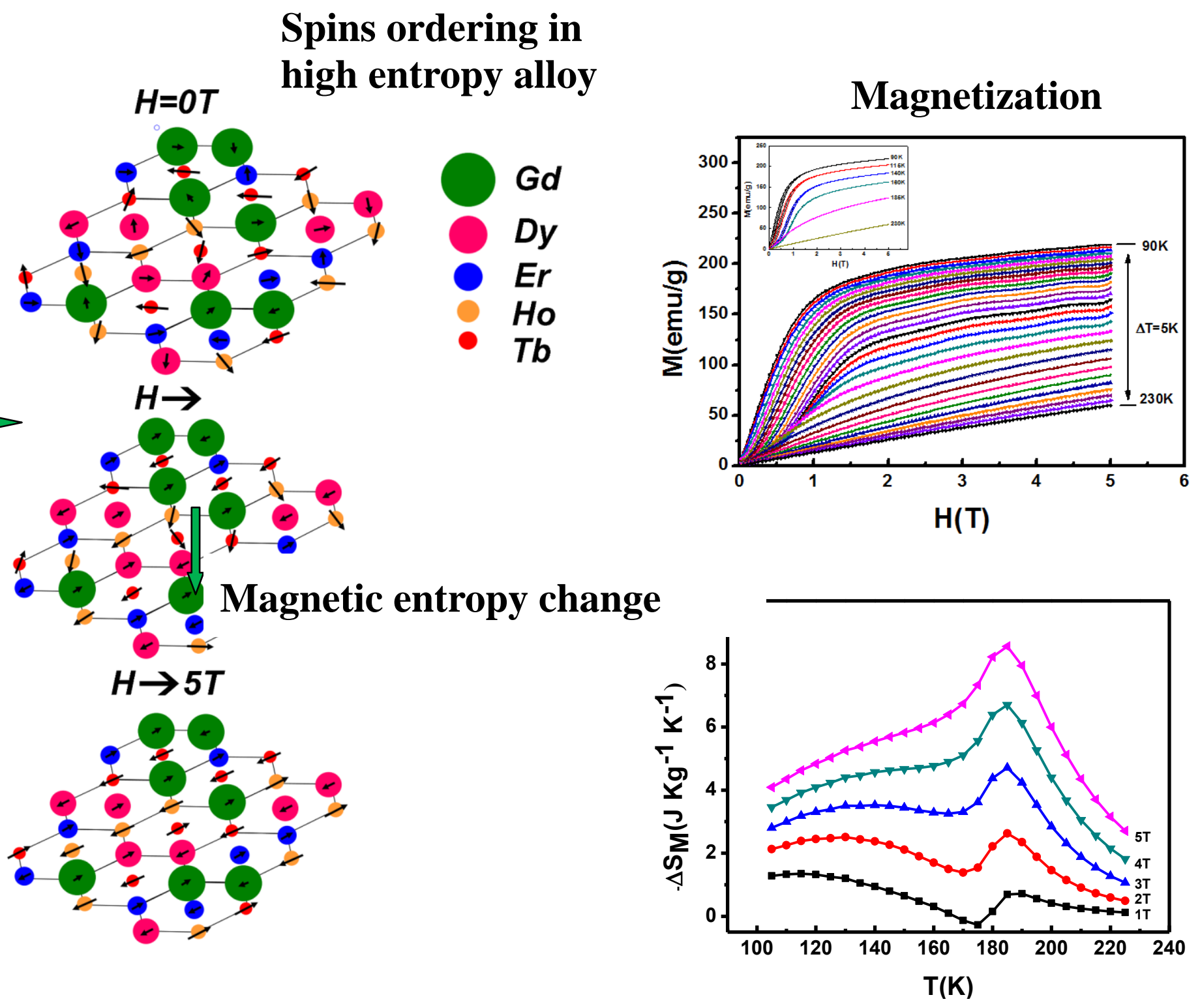

